\title{
EIGENSTRUCTURE OF THE EQUILATERAL TRIANGLE. PART III. THE ROBIN PROBLEM
}

\author{
BRIAN J. MCCARTIN
}

Received 16 June 2003

\begin{abstract}
Lamé's formulas for the eigenvalues and eigenfunctions of the Laplacian on an equilateral triangle under Dirichlet and Neumann boundary conditions are herein extended to the Robin boundary condition. They are shown to form a complete orthonormal system. Various properties of the spectrum and modal functions are explored.
\end{abstract}

2000 Mathematics Subject Classification: 35C05, 35J05, 35P10.

1. Introduction. The eigenstructure of the Laplacian on an equilateral triangle under either Dirichlet or Neumann boundary conditions was explicitly determined by Lamé $[6,7]$ in the context of his studies of heat transfer in polyhedral bodies and then further explored by Pockels [12]. However, Lamé and subsequent researchers such as Pockels did not provide a complete derivation of these formulas but rather simply stated them and then proceeded to show that they satisfied the relevant equation and associated boundary conditions. Such a complete, direct, and elementary derivation of Lamé's formulas has only recently been provided for the Dirichlet problem [11] as well as the Neumann problem [9].

It is the express purpose of the present work to extend this recent work to the much more difficult case of the Robin boundary condition. Lamé [6, 7] presented a partial treatment of this problem when he considered eigenfunctions possessing $120^{\circ}$ rotational symmetry. In all likelihood, Lamé avoided consideration of the complete set of eigenfunctions with Robin boundary conditions because of the attendant complexity of the transcendental equations which so arise. However, armed with the numerical and graphical capabilities of Matlab, we herein study the complete family of Robin eigenfunctions of the Laplacian on an equilateral triangle.

We commence by employing separation of variables in Lamé's natural triangular coordinate system to derive the eigenvalues and eigenfunctions of the Robin problem. An important feature of this derivation is the decomposition into symmetric and antisymmetric modes (eigenfunctions). The problem is then reduced to the solution of a system of transcendental equations which we treat numerically. Surprisingly, all of the modes so determined are expressible as combinations of sines and cosines.

A natural homotopy between Lamé's Neumann and Dirichlet modes is not only exploited in the derivation of the modes but also employed to shed light on the properties of these newly derived modes. Prominent among these considerations are rotational symmetry and modal degeneracy [10]. We resort to a continuity argument in order to 


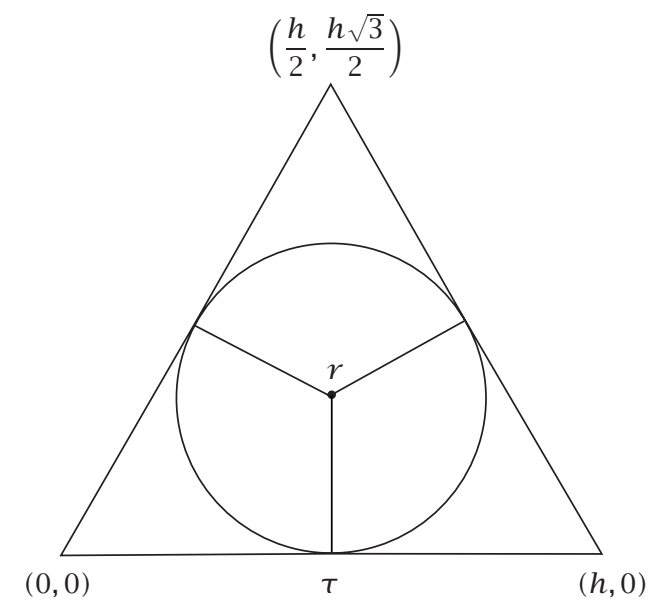

FIGURE 2.1. Equilateral triangle with incircle.

demonstrate orthogonality of these modes. Completeness is then established via an analytic continuation argument relying on the previously published completeness of the Neumann modes [13]. Lastly, knowing the eigenstructure permits us to construct the Robin function [1], and we so do.

2. The Robin eigenproblem for the equilateral triangle. During his investigations into the cooling of a right prism with equilateral triangular base [6, 7], Lamé was led to consider the eigenvalue problem

$$
\begin{aligned}
& \Delta T(x, y)+k^{2} T(x, y)=0, \quad(x, y) \in \tau, \\
& \frac{\partial T}{\partial v}(x, y)+\sigma T(x, y)=0, \quad(x, y) \in \partial \tau,
\end{aligned}
$$

where $\Delta$ is the two-dimensional Laplacian $\partial^{2} / \partial x^{2}+\partial^{2} / \partial y^{2}, \tau$ is the equilateral triangle shown in Figure 2.1, $v$ is its outward pointing normal, and $0 \leq \sigma<+\infty$ is a material parameter. However, he was only able to show that an eigenfunction satisfying (2.1) could be expressed in terms of combinations of sines and cosines when it possesses $120^{\circ}$ rotational symmetry. We will find through the ensuing analysis that all of the eigenfunctions (modes) of this problem are so expressible.

The boundary condition in (2.1) with $\sigma>0$ arises when heat dissipates from a body into a surrounding medium by a combination of convection, radiation, and conduction. It also appears in the study of the vibrational modes of an elastic membrane. If $\sigma$ is allowed to be complex (which is prohibited herein), then the identical problem occurs also in wave propagation in acoustic ducts and electromagnetic waveguides. This is sometimes referred to as a boundary condition of the third kind. However, we will adhere to the more common name of Robin boundary condition even though recent researches $[4,5]$ indicate that this appellation is not historically justified. 


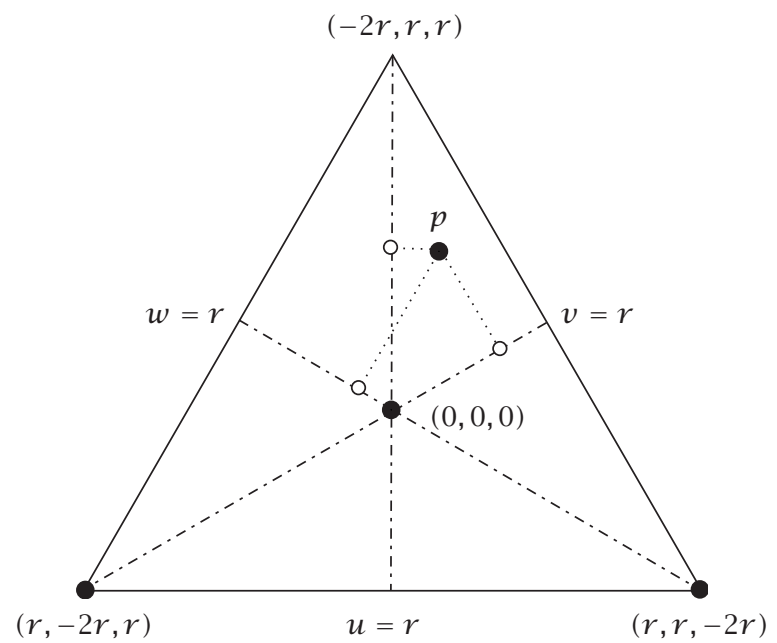

FIGURE 3.1. Triangular coordinate system.

Observe that in (2.1) when $\sigma=0$, we have the Neumann problem [9] while $\sigma \rightarrow+\infty$ yields the Dirichlet problem [11]. Thus, we may profitably view $\sigma$ as a continuation parameter which provides a homotopy between these two well-understood problems. Throughout the ensuing development, we will avail ourselves of this important observation.

3. Triangular coordinate system. Reconsider the equilateral triangle of side $h$ in standard position in Cartesian coordinates $(x, y)$ (Figure 2.1) and define the triangular coordinates $(u, v, w)$ of a point $P$ (Figure 3.1 ) by

$$
\begin{aligned}
& u=r-y, \\
& v=\frac{\sqrt{3}}{2} \cdot\left(x-\frac{h}{2}\right)+\frac{1}{2} \cdot(y-r), \\
& w=\frac{\sqrt{3}}{2} \cdot\left(\frac{h}{2}-x\right)+\frac{1}{2} \cdot(y-r),
\end{aligned}
$$

where $r=h /(2 \sqrt{3})$ is the inradius of the triangle. The coordinates $u, v$, and $w$ may be described as the distances of the triangle center to the projections of the point onto the altitudes, measured positively toward a side and negatively toward a vertex.

Note that Lamé's triangular coordinates satisfy the relation

$$
u+v+w=0
$$

Moreover, the center of the triangle has coordinates $(0,0,0)$ and the three sides of the triangle are given by $u=r, v=r$, and $w=r$, thus simplifying the application of boundary conditions. They are closely related to the barycentric coordinates $(U, V, W)$ 
introduced by Lamé's contemporary Möbius in 1827 (see [3]):

$$
U=\frac{r-u}{3 r}, \quad V=\frac{r-v}{3 r}, \quad W=\frac{r-w}{3 r},
$$

satisfying $U+V+W=1$. These were destined to become the darling of finite element practitioners in the twentieth century.

4. Separation of variables. We now commence with our original, complete, and elementary derivation by introducing the orthogonal coordinates $(\xi, \eta)$ given by

$$
\xi=u, \quad \eta=v-w
$$

Equation (2.1) becomes

$$
\frac{\partial^{2} T}{\partial \xi^{2}}+3 \frac{\partial^{2} T}{\partial \eta^{2}}+k^{2} T=0
$$

Hence, if we seek a separated solution of the form

$$
f(\xi) \cdot g(\eta)
$$

we arrive at

$$
f^{\prime \prime}+\alpha^{2} f=0, \quad g^{\prime \prime}+\beta^{2} g=0, \quad \alpha^{2}+3 \beta^{2}=k^{2} .
$$

Thus, there exist separated solutions of the form

$$
f(u) \cdot g(v-w)
$$

where $f$ and $g$ are trigonometric functions.

Before proceeding any further, we will decompose the sought-after eigenfunction into parts symmetric and antisymmetric about the altitude $v=w$ (see Figure 4.1):

$$
T(u, v, w)=T_{s}(u, v, w)+T_{a}(u, v, w),
$$

where

$$
\begin{aligned}
& T_{s}(u, v, w)=\frac{T(u, v, w)+T(u, w, v)}{2}, \\
& T_{a}(u, v, w)=\frac{T(u, v, w)-T(u, w, v)}{2},
\end{aligned}
$$

henceforth to be dubbed a symmetric/antisymmetric mode, respectively. We next take up the determination of $T_{s}$ and $T_{a}$ separately.

5. Construction of a symmetric mode. As shown elsewhere, a sum of three terms of the form of (4.5) is required to solve either the Neumann [9] or Dirichlet [11] problem. 


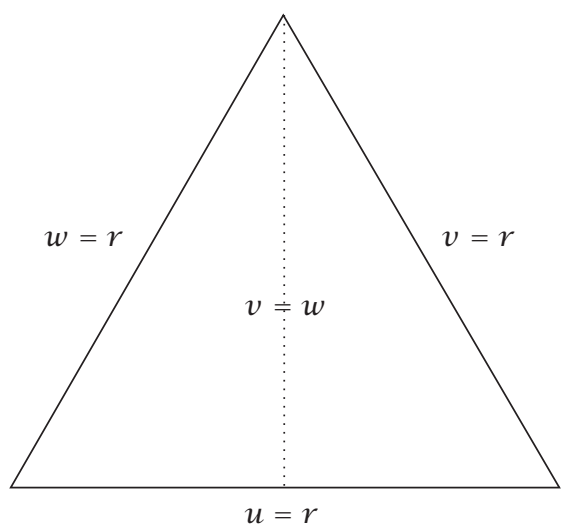

FIGURE 4.1. Modal line of symmetry/antisymmetry.

Hence, we make the ansatz

$$
\begin{aligned}
T_{s}= & \cos \left[\frac{\pi \lambda}{3 r}(u+2 r)-\delta_{1}\right] \cdot \cos \left[\frac{\pi(\mu-v)}{9 r}(v-w)\right] \\
& +\cos \left[\frac{\pi \mu}{3 r}(u+2 r)-\delta_{2}\right] \cdot \cos \left[\frac{\pi(v-\lambda)}{9 r}(v-w)\right] \\
& +\cos \left[\frac{\pi v}{3 r}(u+2 r)-\delta_{3}\right] \cdot \cos \left[\frac{\pi(\lambda-\mu)}{9 r}(v-w)\right],
\end{aligned}
$$

with

$$
\lambda+\nu+\mu=0,
$$

and eigenvalue

$$
k^{2}=\frac{2}{27}\left(\frac{\pi}{r}\right)^{2}\left[\lambda^{2}+\mu^{2}+v^{2}\right]=\frac{4}{27}\left(\frac{\pi}{r}\right)^{2}\left[\mu^{2}+\mu \nu+v^{2}\right] .
$$

As we will see, this symmetric mode never vanishes identically.

Careful perusal of (5.1) now reveals that for $\delta_{1}=\delta_{2}=\delta_{3}=0$, it reduces to a symmetric mode of the Neumann problem [9], while for $\delta_{1}=-3 \pi / 2, \delta_{2}=\pi / 2$, and $\delta_{3}=\pi / 2$, it reduces to a symmetric mode of the Dirichlet problem [11]. Thus, our task amounts to finding values of $\lambda, \mu, v, \delta_{1}, \delta_{2}$, and $\delta_{3}$ so that the Robin boundary condition is satisfied along the periphery of the equilateral triangle. These values are to satisfy the constraints $-3 \pi / 2<\delta_{1} \leq 0,0 \leq \delta_{2}<\pi / 2,0 \leq \delta_{3}<\pi / 2,0 \leq \mu$, and $0 \leq v$, as well as (5.2).

Imposition of the Robin boundary condition along $u=r$ yields

$$
\tan \left(\lambda-\delta_{1}\right)=\frac{3 \sigma r}{\pi \lambda}, \quad \tan \left(\mu-\delta_{2}\right)=\frac{3 \sigma r}{\pi \mu}, \quad \tan \left(v-\delta_{3}\right)=\frac{3 \sigma r}{\pi v},
$$


while the imposition along $v=r$ yields

$$
\tan \left(-\frac{\delta_{2}+\delta_{3}}{2}\right)=\frac{3 \sigma r}{\pi \lambda}, \quad \tan \left(-\frac{\delta_{3}+\delta_{1}}{2}\right)=\frac{3 \sigma r}{\pi \mu}, \quad \tan \left(-\frac{\delta_{1}+\delta_{2}}{2}\right)=\frac{3 \sigma r}{\pi v} .
$$

By symmetry, the boundary condition along $w=r$ will thereby be automatically satisfied.

Introducing the auxiliary variables $L, M$, and $N$ while collecting together these equations produces

$$
\begin{aligned}
& \tan \left(\lambda-\delta_{1}\right)=\tan \left(-\frac{\delta_{2}+\delta_{3}}{2}\right)=\frac{3 \sigma r}{\pi \lambda}=\tan L, \quad-\frac{\pi}{2}<L \leq 0, \\
& \tan \left(\mu-\delta_{2}\right)=\tan \left(-\frac{\delta_{3}+\delta_{1}}{2}\right)=\frac{3 \sigma r}{\pi \mu}=\tan M, \quad 0 \leq M<\frac{\pi}{2}, \\
& \tan \left(v-\delta_{3}\right)=\tan \left(-\frac{\delta_{1}+\delta_{2}}{2}\right)=\frac{3 \sigma r}{\pi v}=\tan N, \quad 0 \leq N<\frac{\pi}{2},
\end{aligned}
$$

and these six equations may in turn be reduced to the solution of the system of three transcendental equations for $L, M$, and $N$ :

$$
\begin{gathered}
{[2 L-M-N-(m+n) \pi] \cdot \tan L=3 \sigma r,} \\
{[2 M-N-L+m \pi] \cdot \tan M=3 \sigma r,} \\
{[2 N-L-M+n \pi] \cdot \tan N=3 \sigma r,}
\end{gathered}
$$

where $m=0,1,2, \ldots$ and $n=m, m+1, \ldots$.

Once $L, M$, and $N$ have been numerically approximated, for example, using Matlab, the parameters of primary interest may then be determined as

$$
\begin{array}{cc}
\delta_{1}=L-M-N, \quad \delta_{2}=-L+M-N, \quad \delta_{3}=-L-M+N, \\
\lambda=\frac{2 L-M-N}{\pi}-m-n, \quad \mu=\frac{2 M-N-L}{\pi}+m, \quad v=\frac{2 N-L-M}{\pi}+n .
\end{array}
$$

For future reference, when $m=n$, we have $M=N, \delta_{2}=\delta_{3}, \mu=\nu$, and $2 \pi \mu=$ $\delta_{2}-\delta_{1}+2 m \pi$.

Of particular interest are the following limits. As $\sigma \rightarrow 0^{+}$, we find that $L, M$, and $N$ each approaches 0 , as do $\delta_{1}, \delta_{2}$, and $\delta_{3}$, and, most significantly, $\lambda \rightarrow-(m+n), \mu \rightarrow m$, and $v \rightarrow n$. In other words, we recover in this limit the Neumann modes. Furthermore, as $\sigma \rightarrow+\infty$, we find that $L \rightarrow-\pi / 2, M \rightarrow \pi / 2, N \rightarrow \pi / 2, \delta_{1} \rightarrow-3 \pi / 2, \delta_{2} \rightarrow \pi / 2$, and $\delta_{3} \rightarrow \pi / 2$, and, most significantly, $\lambda \rightarrow-2-(m+n), \mu \rightarrow m+1$, and $\nu \rightarrow n+1$. In other words, we recover in this limit the Dirichlet modes. Thus, we have successfully fulfilled our original ansatz and thereby constructed a homotopy leading from the symmetric Neumann modes to the symmetric Dirichlet modes. Moreover, we have indexed our symmetric Robin modes $T_{s}^{m, n}$, which are given by (5.1) in correspondence to the symmetric Neumann modes with the result that as $\sigma$ ranges from 0 to $+\infty$, the $(m, n)$ symmetric Neumann mode "morphs" continuously (in fact, analytically) into the $(m+1, n+1)$ symmetric Dirichlet mode.

Figure 5.1 shows the plane wave $(0,0)$ mode morphing into the fundamental $(1,1)$ Dirichlet mode. Figure 5.2 displays the $(0,1)$ symmetric mode. 

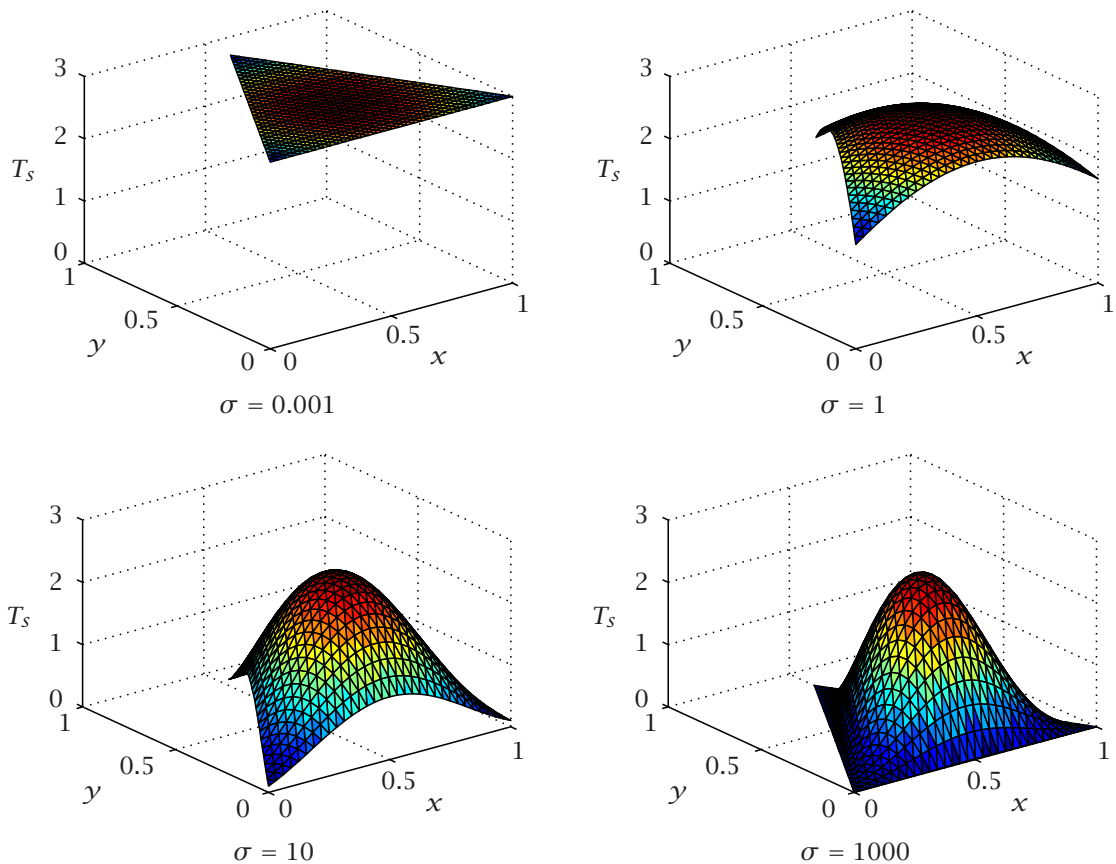

Figure 5.1. The $(0,0)$ symmetric mode.
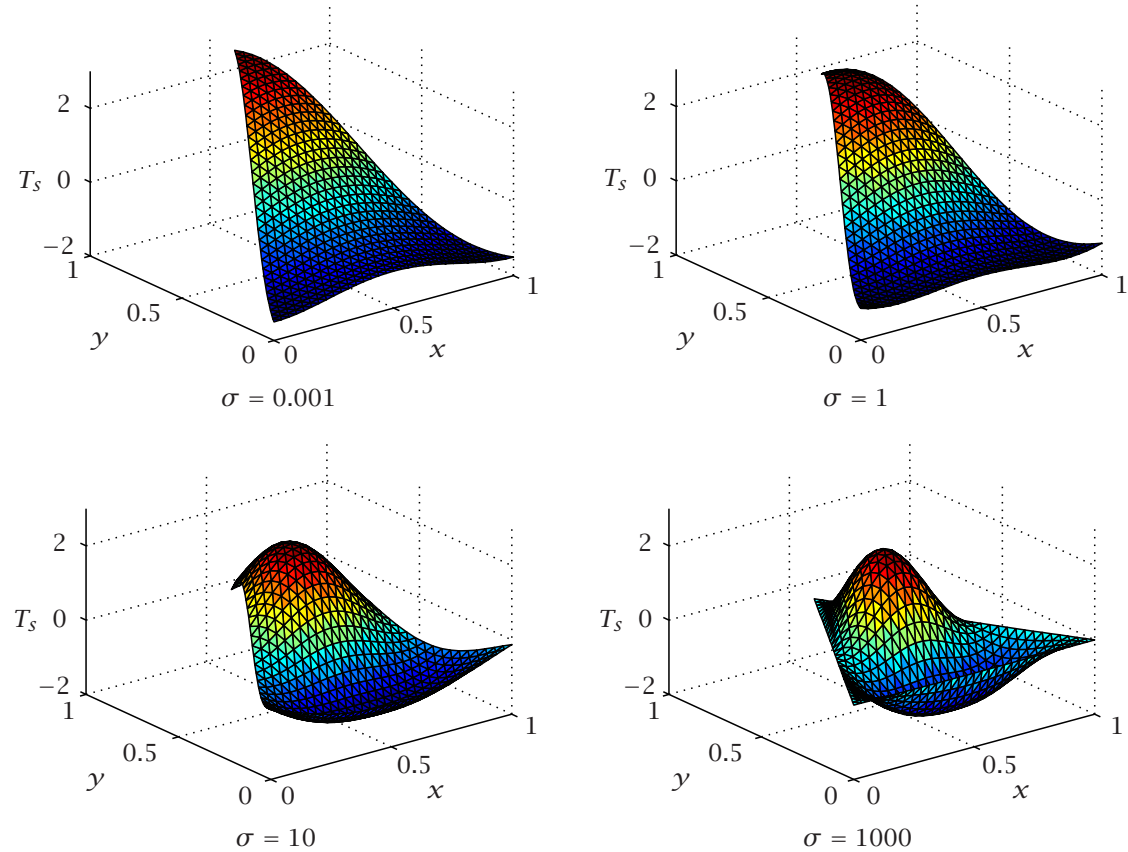

Figure 5.2. The $(0,1)$ symmetric mode. 

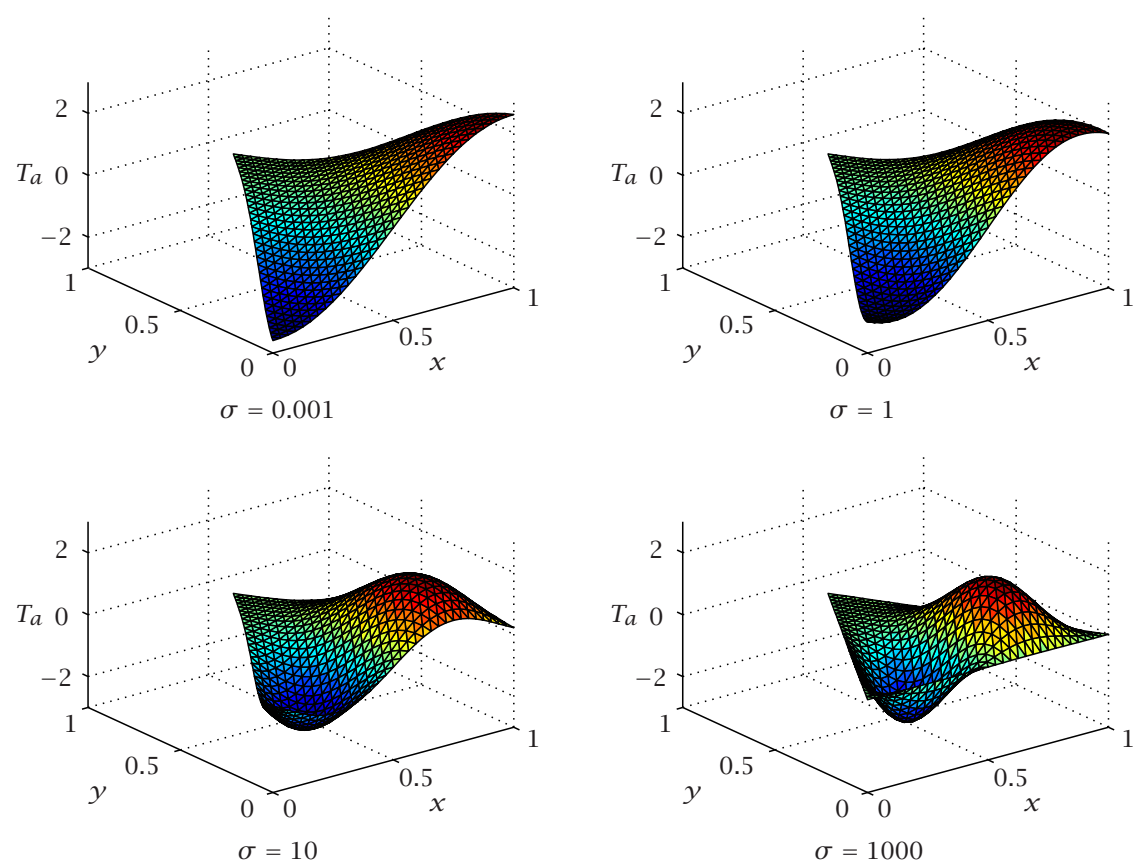

FIGURE 6.1. The $(0,1)$ antisymmetric mode.

6. Construction of an antisymmetric mode. A parallel development is possible for the determination of an antisymmetric mode. In light of the oddness of $T_{a}$ as a function of $v-w$, we commence with an ansatz of the form

$$
\begin{aligned}
T_{a}= & \cos \left[\frac{\pi \lambda}{3 r}(u+2 r)-\delta_{1}\right] \cdot \sin \left[\frac{\pi(\mu-v)}{9 r}(v-w)\right] \\
& +\cos \left[\frac{\pi \mu}{3 r}(u+2 r)-\delta_{2}\right] \cdot \sin \left[\frac{\pi(v-\lambda)}{9 r}(v-w)\right] \\
& +\cos \left[\frac{\pi v}{3 r}(u+2 r)-\delta_{3}\right] \cdot \sin \left[\frac{\pi(\lambda-\mu)}{9 r}(v-w)\right]
\end{aligned}
$$

Once again,

$$
\begin{aligned}
\lambda+\mu+\nu & =0, \\
k^{2}=\frac{2}{27}\left(\frac{\pi}{r}\right)^{2}\left[\lambda^{2}+\mu^{2}+\nu^{2}\right] & =\frac{4}{27}\left(\frac{\pi}{r}\right)^{2}\left[\mu^{2}+\mu \nu+v^{2}\right] .
\end{aligned}
$$

However, this antisymmetric mode may vanish identically.

Equations (5.4), (5.5), (5.6), (5.7), and (5.8) still hold so that, for a given $m$ and $n$, $\left\{\lambda, \mu, \nu, \delta_{1}, \delta_{2}, \delta_{3}\right\}$ are the same for the symmetric, $T_{s}^{m, n}$, and antisymmetric, $T_{a}^{m, n}$, modes which are given by (6.1). In particular, the comments made above concerning indexing modes and "mode-morphing" also apply here. Figure 6.1 displays the $(0,1)$ antisymmetric mode. 
7. Modal properties. In what follows, it will be convenient to have the following alternative representations of our Robin modes:

$$
\begin{aligned}
T_{s}^{m, n}=\frac{1}{2}\{ & \cos \left[\frac{2 \pi}{9 r}(\lambda u+\mu v+v w+3 \lambda r)-\delta_{1}\right] \\
& +\cos \left[\frac{2 \pi}{9 r}(\nu u+\mu v+\lambda w+3 v r)-\delta_{3}\right] \\
& +\cos \left[\frac{2 \pi}{9 r}(\mu u+v v+\lambda w+3 \mu r)-\delta_{2}\right] \\
& +\cos \left[\frac{2 \pi}{9 r}(\mu u+\lambda v+v w+3 \mu r)-\delta_{2}\right] \\
& +\cos \left[\frac{2 \pi}{9 r}(\nu u+\lambda v+\mu w+3 v r)-\delta_{3}\right] \\
& \left.+\cos \left[\frac{2 \pi}{9 r}(\lambda u+v v+\mu w+3 \lambda r)-\delta_{1}\right]\right\}, \\
T_{a}^{m, n}=\frac{1}{2}\{ & \sin \left[\frac{2 \pi}{9 r}(\lambda u+\mu v+v w+3 \lambda r)-\delta_{1}\right] \\
& -\sin \left[\frac{2 \pi}{9 r}(v u+\mu v+\lambda w+3 v r)-\delta_{3}\right] \\
& +\sin \left[\frac{2 \pi}{9 r}(\mu u+v v+\lambda w+3 \mu r)-\delta_{2}\right] \\
& -\sin \left[\frac{2 \pi}{9 r}(\mu u+\lambda v+v w+3 \mu r)-\delta_{2}\right] \\
& +\sin \left[\frac{2 \pi}{9 r}(v u+\lambda v+\mu w+3 v r)-\delta_{3}\right] \\
& \left.-\sin \left[\frac{2 \pi}{9 r}(\lambda u+v v+\mu w+3 \lambda r)-\delta_{1}\right]\right\},
\end{aligned}
$$

obtained from (5.1) and (6.1), respectively, by the application of appropriate trigonometric identities.

We may reduce the collection of antisymmetric Robin modes through the following observation.

THEOREM 7.1. (i) The mode $T_{s}^{m, n}$ never vanishes identically.

(ii) The mode $T_{a}^{m, n}$ vanishes identically if and only if $m=n$.

Proof. (i) Note that a symmetric mode is identically zero if and only if it vanishes along the line of symmetry $v=w$ since the only function, both symmetric and antisymmetric, is the zero function. Along $v=w$,

$$
\begin{aligned}
T_{s}^{m, n}= & \cos \left[\frac{\pi(\mu+v)}{3 r}(u+2 r)-\delta_{1}\right]+\cos \left[\frac{\pi \mu}{3 r}(u+2 r)-\delta_{2}\right] \\
& +\cos \left[\frac{\pi v}{3 r}(u+2 r)-\delta_{3}\right]
\end{aligned}
$$

which cannot vanish identically for $-2 r \leq u \leq r$. 


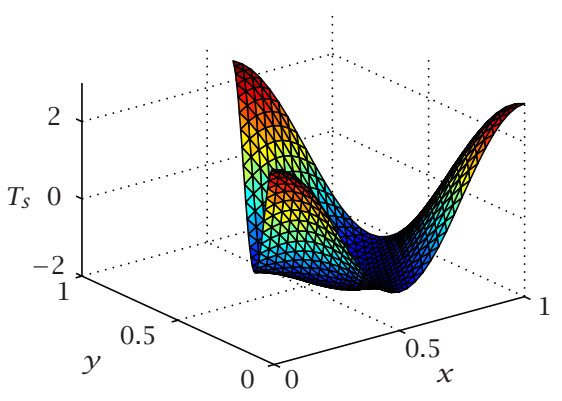

$\sigma=0.001$

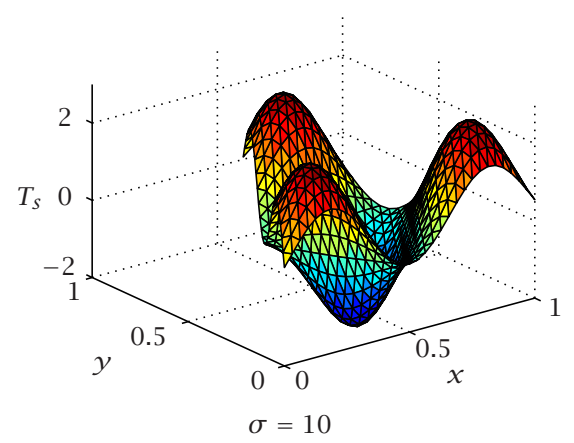

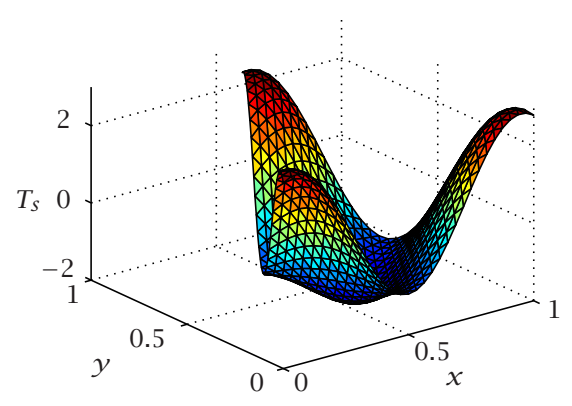

$\sigma=1$

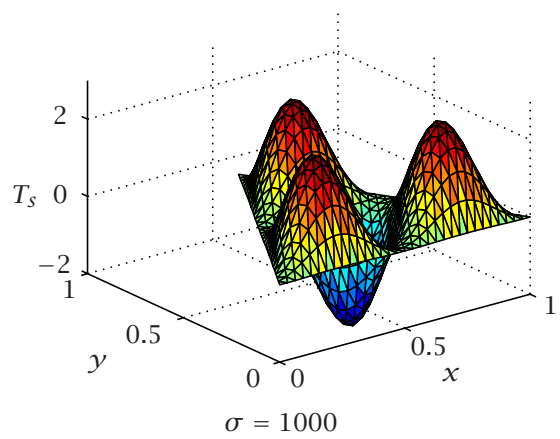

FiguRE 7.1. The $(1,1)$ symmetric mode.

(ii) Note that an antisymmetric mode is identically zero if and only if its normal derivative vanishes along the line of symmetry $v=w$ since the only function, both antisymmetric and symmetric, is the zero function. Along $v=w$,

$$
\begin{aligned}
\frac{\partial T_{a}^{m, n}}{\partial(v-w)}= & \frac{\pi(\mu-v)}{9 r} \cos \left[\frac{\pi(\mu+v)}{3 r}(u+2 r)+\delta_{1}\right] \\
& +\frac{\pi(\mu+2 v)}{9 r} \cos \left[\frac{\pi \mu}{3 r}(u+2 r)-\delta_{2}\right] \\
& -\frac{\pi(2 \mu+v)}{9 r} \cos \left[\frac{\pi v}{3 r}(u+2 r)-\delta_{3}\right] .
\end{aligned}
$$

This equals zero if and only if $m=n$.

Hence, our system of eigenfunctions is $\left\{T_{s}^{m, n}(n \geq m) ; T_{a}^{m, n}(n>m)\right\}$. Figure 7.1 shows the $(1,1)$ mode whereas the symmetric and antisymmetric $(0,2)$ modes are displayed in Figures 7.2 and 7.3, respectively, while the symmetric and antisymmetric $(1,2)$ modes are displayed in Figures 7.4 and 7.5 , respectively. 


$$
\begin{aligned}
& \text { D } k \\
& \text { a } n \\
& \text { a } \\
& \text { A }
\end{aligned}
$$



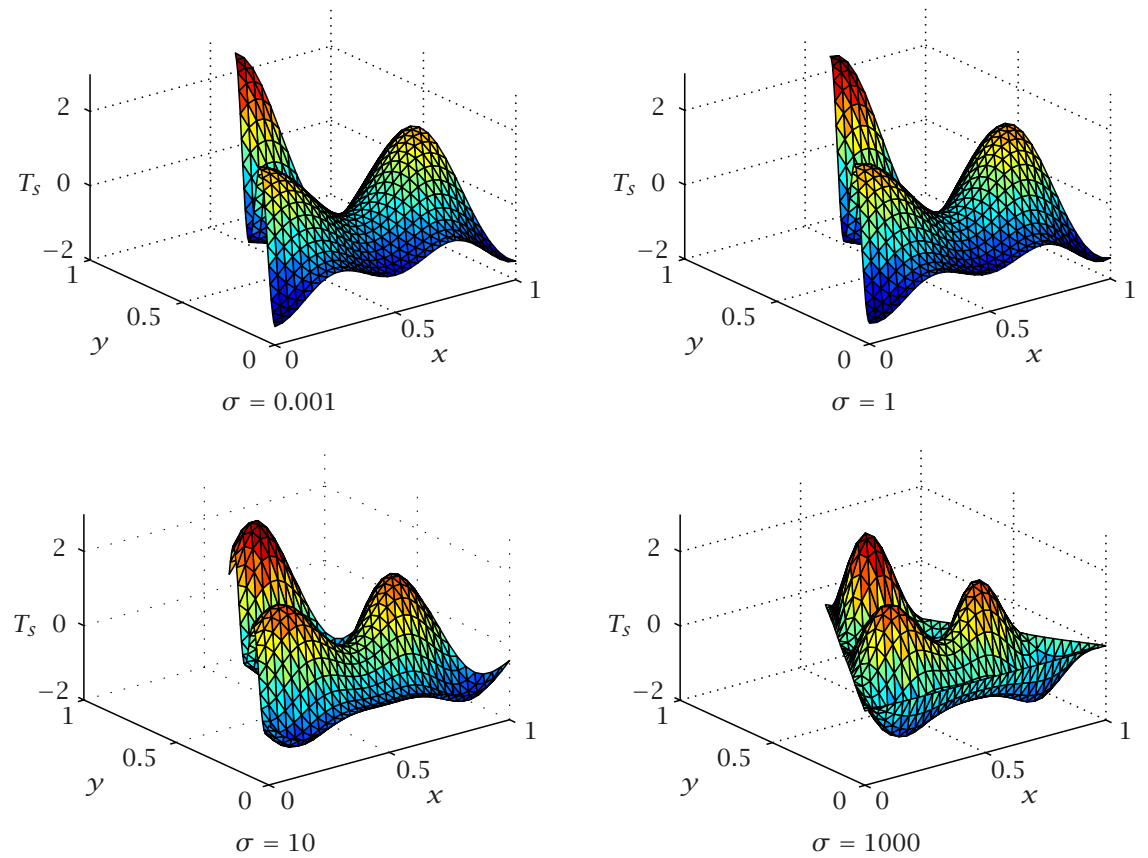

FIGURE 7.4. The $(1,2)$ symmetric mode.
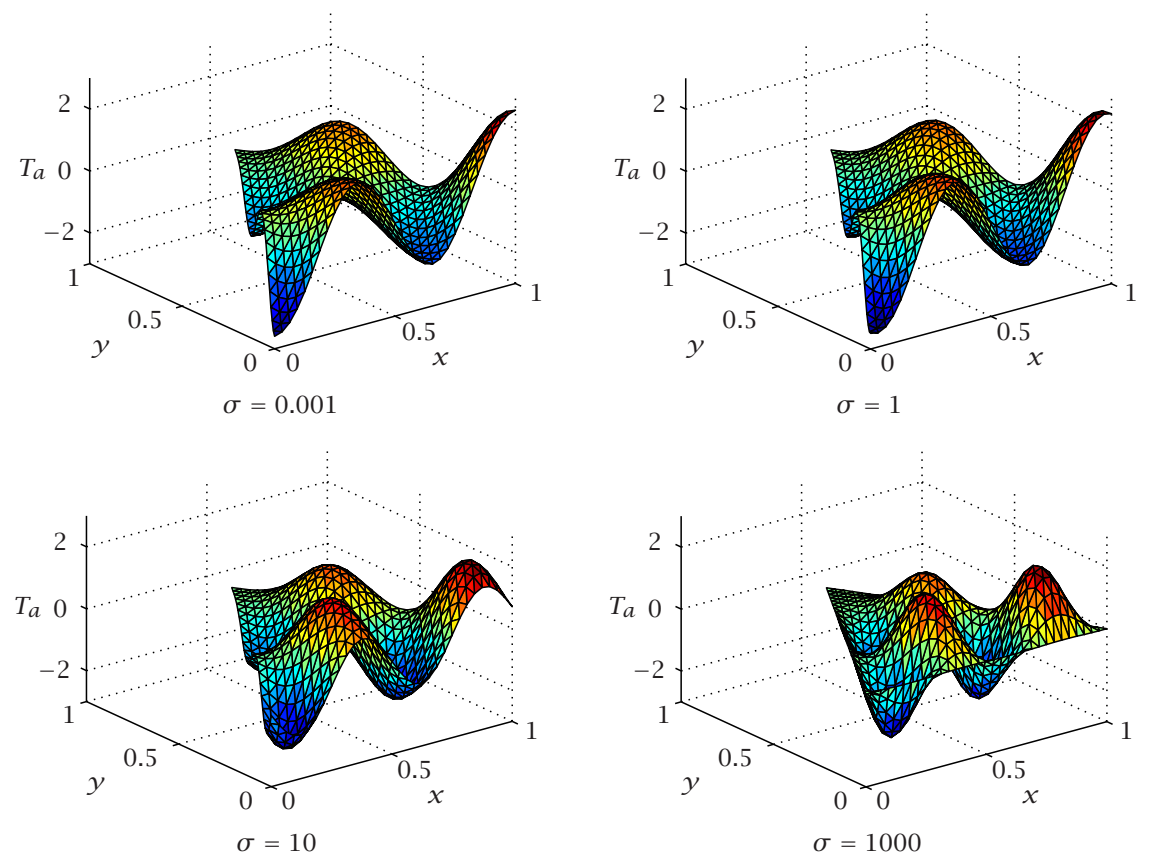

FIgURE 7.5 . The $(1,2)$ antisymmetric mode. 
We next give the case $m=n$ further consideration. Recall that we have just determined that $T_{a}^{m, m} \equiv 0$. Furthermore, in this case, we may combine the terms of (7.1) to yield

$$
\begin{aligned}
T_{s}^{m, m}= & \cos \left[\frac{2 \pi \mu}{3 r}(r-u)-\delta_{2}\right] \\
& +\cos \left[\frac{2 \pi \mu}{3 r}(r-v)-\delta_{2}\right] \\
& +\cos \left[\frac{2 \pi \mu}{3 r}(r-w)-\delta_{2}\right],
\end{aligned}
$$

which clearly illustrates that any permutation of $(u, v, w)$ leaves $T_{s}^{m, m}$ invariant. This is manifested geometrically in the invariance of $T_{s}^{m, m}$ under a $120^{\circ}$ rotation about the triangle center (see Figure 7.1). This invariance will henceforth be termed rotational symmetry.

Moreover, the modes $T_{s}^{m, m}$ are not the only ones that are rotationally symmetric.

THEOREM 7.2. (i) The mode $T_{s}^{m, n}$ is rotationally symmetric if and only if

$$
m \equiv n(\equiv l)(\bmod 3) .
$$

(ii) The mode $T_{a}^{m, n}$ is rotationally symmetric if and only if $m \equiv n(\equiv l)(\bmod 3)$.

Proof. (i) The mode $T_{s}^{m, n}$ is rotationally symmetric if and only if it is symmetric about the line $v=u$. This can occur if and only if the normal derivative $\partial T_{s}^{m, n} / \partial v$ vanishes there. Thus, we require that

$$
\begin{aligned}
\left.\frac{\partial T_{s}^{m, n}}{\partial(v-u)}\right|_{v=u}=-\frac{1}{4}\{ & (2 \mu+v) \sin \left[\frac{2 \pi}{3 r}(-v u-(\mu+v) r)-\delta_{1}\right] \\
& +(\mu-v) \sin \left[\frac{2 \pi}{3 r}((\mu+v) u+v r)-\delta_{3}\right] \\
& -(\mu-v) \sin \left[\frac{2 \pi}{3 r}((\mu+v) u+\mu r)-\delta_{2}\right] \\
& -(2 \mu+v) \sin \left[\frac{2 \pi}{3 r}(-v u+\mu r)-\delta_{2}\right] \\
& -(\mu+2 v) \sin \left[\frac{2 \pi}{3 r}(-\mu u+v r)-\delta_{3}\right] \\
& \left.+(\mu+2 v) \sin \left[\frac{2 \pi}{3 r}(-\mu u-(\mu+v) r)-\delta_{1}\right]\right\}=0,
\end{aligned}
$$

derived from (7.1). These terms cancel pairwise if and only if (7.6) holds. 

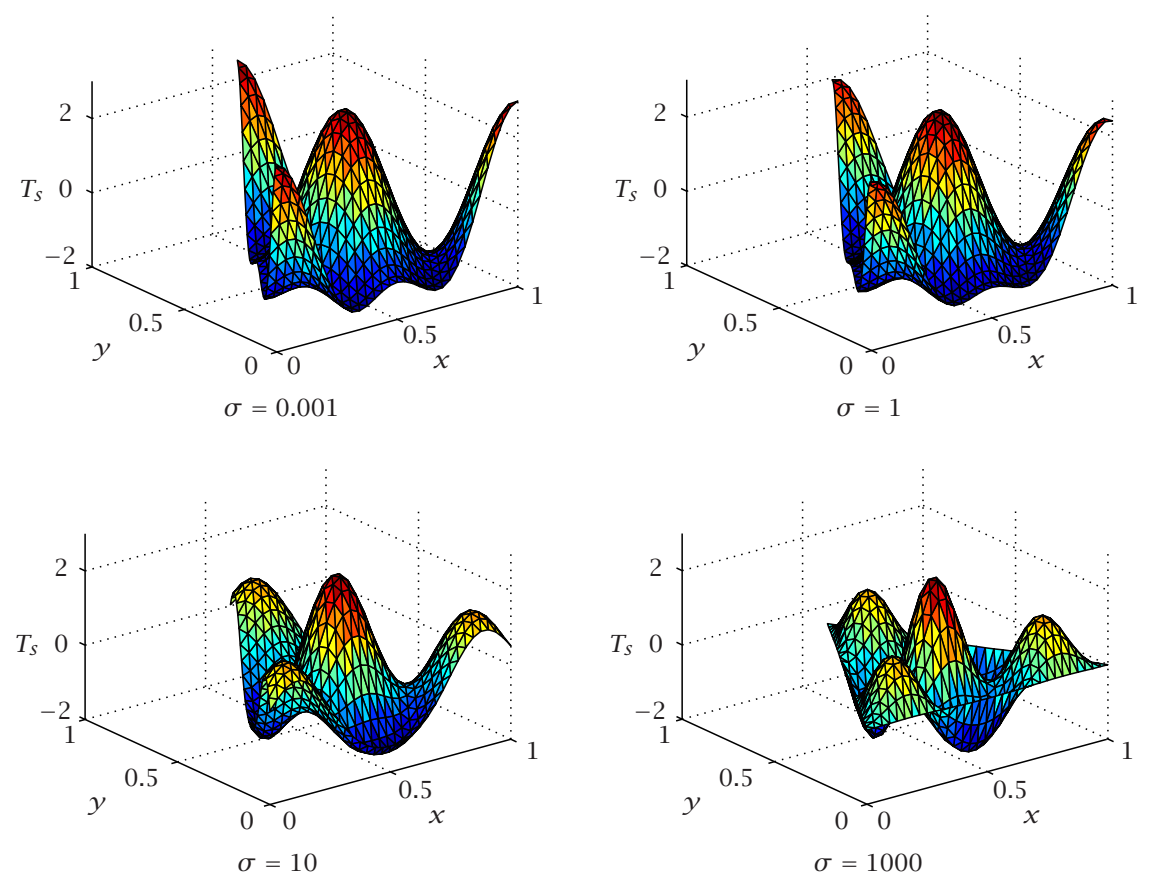

FIGURE 7.6. The $(0,3)$ symmetric mode.

(ii) The mode $T_{a}^{m, n}$ is rotationally symmetric if and only if it is antisymmetric about the line $v=u$. This can occur if and only if $T_{a}^{m, n}$ vanishes there. Thus, we require that

$$
\begin{aligned}
\left.T_{a}^{m, n}\right|_{v=u}=\frac{1}{2}\{ & \sin \left[\frac{2 \pi}{3 r}(-v u-(\mu+v) r)-\delta_{1}\right] \\
& -\sin \left[\frac{2 \pi}{3 r}((\mu+v) u+v r)-\delta_{3}\right] \\
& +\sin \left[\frac{2 \pi}{3 r}((\mu+v) u+\mu r)-\delta_{2}\right] \\
& -\sin \left[\frac{2 \pi}{3 r}(-v u+\mu r)-\delta_{2}\right] \\
& +\sin \left[\frac{2 \pi}{3 r}(-\mu u+v r)-\delta_{3}\right] \\
& \left.-\sin \left[\frac{2 \pi}{3 r}(-\mu u-(\mu+v) r)-\delta_{1}\right]\right\}=0,
\end{aligned}
$$

derived from (7.2). These terms cancel pairwise if and only if (7.6) holds.

This is illustrated in Figures 7.6 and 7.7 which display the symmetric and antisymmetric $(0,3)$ modes, respectively. 

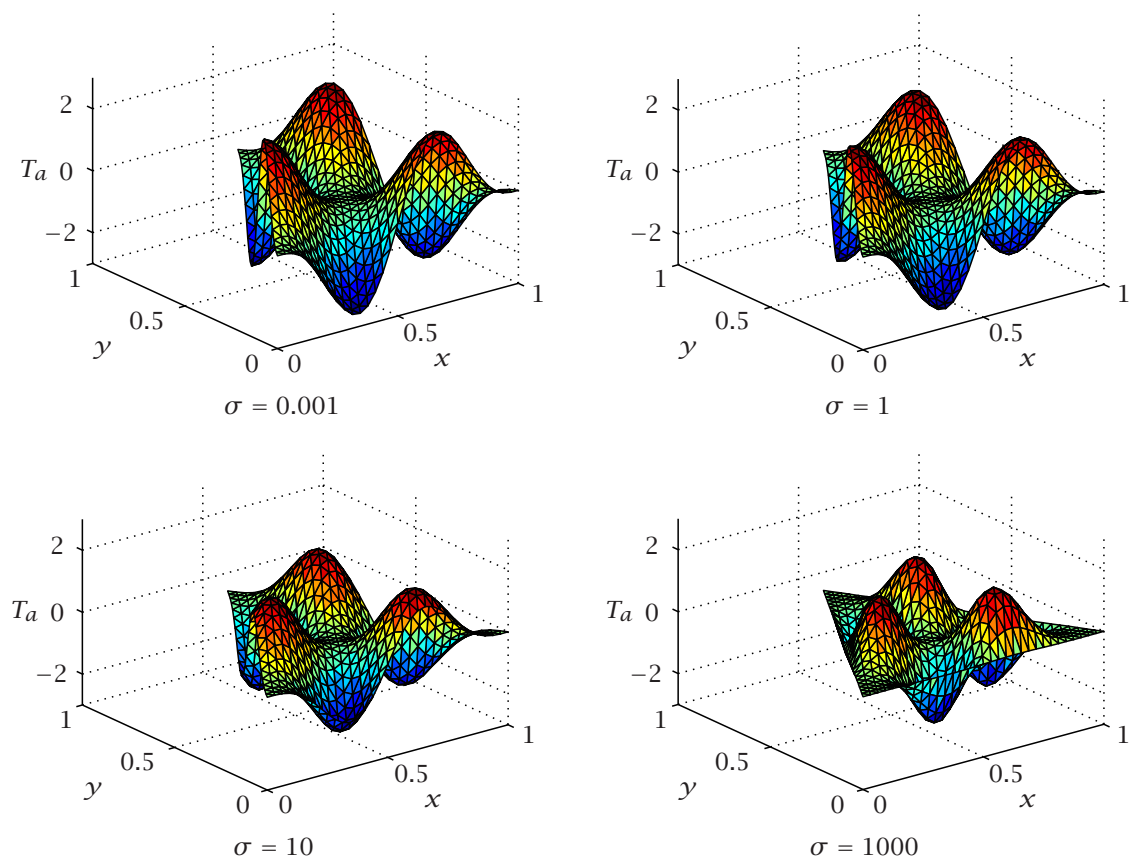

FIgURE 7.7 . The $(0,3)$ antisymmetric mode.

8. Spectral properties. The modal frequencies $f_{m, n}$ are proportional to the square root of the eigenvalues given by (5.3). Hence, we have

$$
f_{m, n} \propto \frac{4 \pi}{3 h} \chi, \quad \chi^{2}:=\mu^{2}+\mu \nu+v^{2} .
$$

Thus, the spectral structure of the equilateral triangle hinges upon the properties of the spectral parameter $\chi^{2}$.

This spectral parameter is shown for the first 29 modes in Figure 8.1 as $\sigma$ ranges from 0 to $+\infty$. The left side corresponds to the Neumann modes $(\sigma=0)$ and the right side corresponds to the Dirichlet modes $(\sigma=+\infty)$. Thus, this figure graphically displays the homotopy relating these two well-understood eigenvalue problems.

The monotonicity of these curves is apparent and easily established from the identity

$$
\frac{d \chi^{2}}{d \sigma}=(2 \mu+v) \frac{d \mu}{d \sigma}+(\mu+2 v) \frac{d v}{d \sigma} \geq 0
$$

since $(\mu, v)$ increase monotonically from $(m, n)$ to $(m+1, n+1)$ as $\sigma$ varies from 0 to $+\infty$.

Since $T_{s}^{m, n}$ and $T_{a}^{m, n}$ both correspond to the same frequency $f_{m, n}$ given by (8.1), it follows that all eigenvalues corresponding to $m \neq n$ have multiplicity equal to at least two. However, this modal degeneracy, as it is known in the engineering literature, extends also to the case $m=n$. Modal degeneracy is manifested in Figure 8.1 as an 


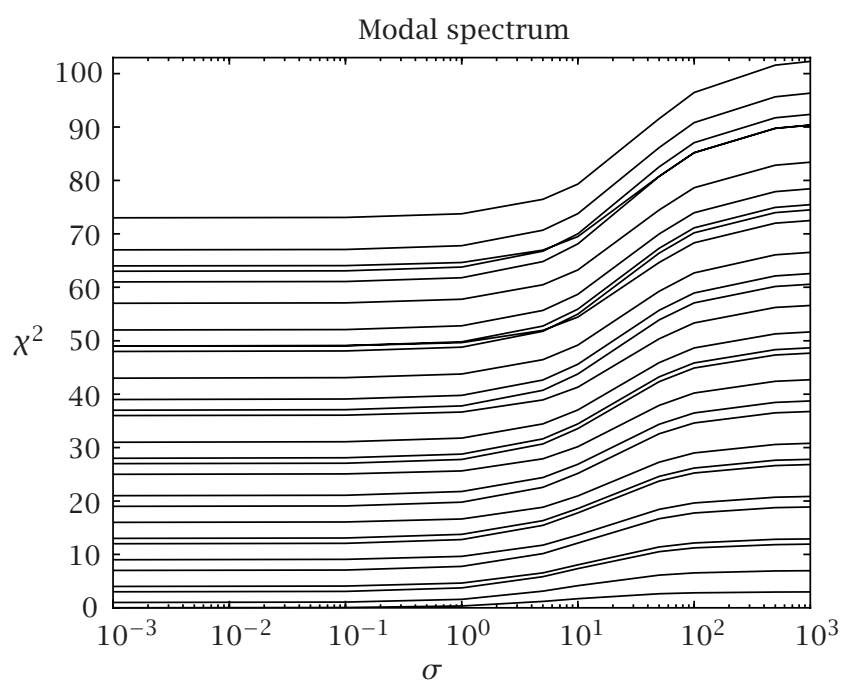

FIGURE 8.1. Spectral parameter.

intersection of two modal curves. For the Dirichlet and Neumann problems, numbertheoretic techniques permit a comprehensive treatment of such spectral multiplicity [10].

However, for the Robin problem, $\mu$ and $v$ are not integers and such techniques fail. At the present time, no general results are available and one must resort to perusal of Figure 8.1 in order to locate modal degeneracies for $0<\sigma<+\infty$. All one can say with certainty is that if $\left(m_{1}, n_{1}\right)$ and $\left(m_{2}, n_{2}\right)$ are modal indices satisfying the inequalities

$$
0<\left(m_{2}^{2}+m_{2} n_{2}+n_{2}^{2}\right)-\left(m_{1}^{2}+m_{1} n_{1}+n_{1}^{2}\right)<3\left[\left(m_{1}+n_{1}\right)-\left(m_{2}+n_{2}\right)\right],
$$

then the corresponding modal curves must intersect for some value of $\sigma$ which will thereby generate a corresponding modal degeneracy.

9. Orthogonality. By Rellich's theorem [8], eigenfunctions corresponding to distinct eigenvalues are guaranteed to be orthogonal. Also, a symmetric mode and an antisymmetric mode are automatically orthogonal. However, as we discovered above, the multiplicity of the eigenvalues given by (5.3) is quite a complicated matter. Thus, we invoke the following continuity argument in order to confirm the orthogonality of our collection of eigenfunctions $\left\{T_{s}^{m, n}(n \geq m) ; T_{a}^{m, n}(n>m)\right\}$.

Suppose that $f$ and $g$ are eigenfunctions of like parity that share an eigenvalue, $k^{2}$, for some fixed value of $\sigma=\hat{\sigma}$. This corresponds to an intersection of two spectral curves in Figure 8.1. For $\sigma$ in the neighborhood of $\hat{\sigma}$, Rellich's theorem guarantees that $\langle f, g\rangle=$ $\iint_{\tau} f g d A=0$. Thus, by continuity, $\langle f, g\rangle=0$ for $\sigma=\hat{\sigma}$ and the orthogonality of our full collection of Robin modes is assured. Note that this same continuity argument may be employed to demonstrate the orthogonality of the Robin eigenfunctions for other differential operators on other domains. 
10. Completeness. It is not a priori certain that the collection of eigenfunctions $\left\{T_{s}^{m, n}, T_{a}^{m, n}\right\}$ constructed above is complete. For domains which are the Cartesian product of intervals in an orthogonal coordinate system, such as rectangles and annuli, completeness of the eigenfunctions formed from products of one-dimensional counterparts has been established [15]. Since the equilateral triangle is not such a domain, we must employ other devices in order to establish completeness.

We will utilize an analytic continuation argument which hinges upon the previously established completeness of the Neumann modes [13]. The homotopy between the Neumann and Dirichlet modes that we have established above guarantees a unique branch leading from each of the Neumann modes to its corresponding Dirichlet mode. Likewise, for any $0<\sigma<\infty$, we may trace out a branch from any mode leading back to a Neumann mode as $\sigma \rightarrow 0^{+}$.

Suppose, for the sake of argument, that the collection of Robin modes constructed above is not complete for some $0<\sigma=\hat{\sigma}<\infty$. Then, let $u(x, y ; \hat{\sigma})$ be a mode that is not contained in our collection. As we have a selfadjoint operator, there exist $\ell$ analytic branches emanating from this point in Hilbert space where $\ell$ is the multiplicity of $k^{2}(\hat{\sigma})$ [2]. Denote any of these branches, analytically continued back to $\sigma=0$ as $u(x, y ; \sigma)$. Since we know that the collection of Neumann modes is complete, this branch must at some point, $\sigma=\sigma^{*}$, coalesce with a branch emanating from one of our Robin modes.

However, as we now show, the analytic dependence of $u(x, y ; \sigma)$ upon $\sigma$ prohibits such a bifurcation at $\sigma=\sigma^{*}$. To see this, let

$$
\Delta u+k^{2} u=0, \quad(x, y) \in \tau, \quad \frac{\partial u}{\partial v}+\sigma u=0, \quad(x, y) \in \partial \tau .
$$

Then

$$
\begin{aligned}
u(x, y ; \sigma)= & u\left(x, y ; \sigma^{*}\right)+u^{\prime}\left(x, y ; \sigma^{*}\right) \cdot\left(\sigma-\sigma^{*}\right) \\
& +u^{\prime \prime}\left(x, y ; \sigma^{*}\right) \cdot \frac{\left(\sigma-\sigma^{*}\right)^{2}}{2}+\cdots,
\end{aligned}
$$

where $u^{\prime}:=\partial u / \partial \sigma$ and each of the correction terms in the Taylor series is orthogonal to the eigenspace of $k^{2}\left(\sigma^{*}\right)$.

Each of the Taylor coefficients satisfies the boundary value problem

$$
\begin{gathered}
\Delta u^{(n)}\left(x, y ; \sigma^{*}\right)+k^{2}\left(\sigma^{*}\right) u^{(n)}\left(x, y ; \sigma^{*}\right)=0, \quad(x, y) \in \tau, \\
\frac{\partial u^{(n)}}{\partial v}\left(x, y ; \sigma^{*}\right)+\sigma^{*} u^{(n)}\left(x, y ; \sigma^{*}\right)=-n u^{(n-1)}\left(x, y ; \sigma^{*}\right), \quad(x, y) \in \partial \tau,
\end{gathered}
$$

which may be solved recursively and uniquely for $u^{\prime}, u^{\prime \prime}, \ldots, u^{(n)}, \ldots$ since they are each orthogonal to the eigenspace of $k^{2}$. Thus, $u(x, y ; \sigma)$ is uniquely determined and bifurcation cannot transpire. Consequently, our collection of Robin modes is indeed complete. Note that this same analytic continuation argument may be invoked to establish the completeness of Robin eigenfunctions for other differential operators on other domains once the completeness of the corresponding Neumann eigenfunctions has been certified. 
11. Robin function. Using (6.1) and (7.1), we may define the orthonormal system of eigenfunctions

$$
\begin{aligned}
& \phi_{s}^{m, n}=\frac{T_{s}^{m, n}}{\left\|T_{s}^{m, n}\right\|} \quad(m=0,1,2, \ldots ; n=m, \ldots), \\
& \phi_{a}^{m, n}=\frac{T_{a}^{m, n}}{\left\|T_{a}^{m, n}\right\|} \quad(m=0,1,2, \ldots ; n=m+1, \ldots),
\end{aligned}
$$

together with their corresponding eigenvalues

$$
\lambda_{m, n}=\frac{4 \pi^{2}}{27 r^{2}}\left(\mu^{2}+\mu \nu+v^{2}\right) \quad(m=0,1,2, \ldots ; n=m, \ldots) .
$$

Green's function [14] for the Laplacian with Robin boundary conditions (the Robin function [1]) on an equilateral triangle is then constructed as

$$
\begin{aligned}
G\left(x, y ; x^{\prime}, y^{\prime}\right) & \\
= & \sum_{m=1}^{\infty} \frac{\phi_{s}^{m, m}(x, y) \phi_{s}^{m, m}\left(x^{\prime}, y^{\prime}\right)}{\lambda_{m, m}} \\
& +\sum_{m=0}^{\infty} \sum_{n=m+1}^{\infty} \frac{\phi_{s}^{m, n}(x, y) \phi_{s}^{m, n}\left(x^{\prime}, y^{\prime}\right)+\phi_{a}^{m, n}(x, y) \phi_{a}^{m, n}\left(x^{\prime}, y^{\prime}\right)}{\lambda_{m, n}} .
\end{aligned}
$$

This may be employed in the usual fashion to solve the corresponding nonhomogeneous boundary value problem [1].

12. Conclusion. In the foregoing, we have filled a prominent gap in the applied mathematical literature by providing a complete elementary derivation of the extension of Lamé's formulas for the eigenfunctions of the equilateral triangle to Robin boundary conditions. In addition to its innate mathematical interest, this problem is of practical interest as it relates to the calibration of numerical algorithms for approximating the eigenvalues of the Laplacian upon triangulated domains.

In addition, we have established the orthonormality and completeness of this collection of eigenfunctions using the simplest of mathematical tools. Furthermore, we have made an extensive investigation of the properties of the spectrum and modes. Lastly, the Robin function has been specified.

We close with the observation that the above development is inherently dependent upon the constancy of $\sigma$. If the eigenfunctions for variable $\sigma$ were trigonometric, then that would imply that the corresponding eigenfunctions for the problem with Dirichlet/Neumann conditions along two sides of the triangle and a Neumann/Dirichlet condition, respectively, along the third side were also trigonometric. This would violate theorems to the contrary established in $[9,11]$.

ACKNOWLEDGMENTs. The author thanks Mrs. Barbara A. McCartin for her indispensable aid in constructing the figures. This paper is dedicated with respect, admiration, and gratitude to my thesis advisor, Professor Antony Jameson (Courant, Princeton, Stanford). 


\section{REFERENCES}

[1] G. F. D. Duff, Partial Differential Equations, Mathematical Expositions, no. 9, University of Toronto Press, Toronto, 1956.

[2] K. O. Friedrichs, Perturbation of Spectra in Hilbert Space, American Mathematical Society, Rhode Island, 1965.

[3] J. Gray, Möbius's geometrical mechanics, Möbius and His Band (J. Fauvel et al., ed.), Oxford University Press, New York, 1993.

[4] K. Gustafson and T. Abe, The third boundary condition-was it Robin's? Math. Intelligencer 20 (1998), no. 1, 63-71.

[5] _ (Victor) Gustave Robin: 1855-1897, Math. Intelligencer 20 (1998), no. 2, 47-53.

[6] G. Lamé, Mémoire sur la propagation de la chaluer dans les polyèdres, Journal de l'École Polytechnique 22 (1833), 194-251 (French).

[7] _ Leçons sur la Théorie Analytique de la Chaleur, Mallet-Bachelier, Paris, 1861 (French).

[8] C. R. MacCluer, Boundary Value Problems and Orthogonal Expansions. Physical Problems from a Sobolev Viewpoint, IEEE Press, New Jersey, 1994.

[9] B. J. McCartin, Eigenstructure of the equilateral triangle. II. The Neumann problem, Math. Probl. Eng. 8 (2002), no. 6, 517-539.

[10] _ Modal degeneracy in equilateral triangular waveguides, J. Electromagn. Waves Appl. 16 (2002), no. 7, 943-956.

[11]__ Eigenstructure of the Equilateral Triangle, Part I: The Dirichlet Problem, SIAM Rev. 45 (2003), no. 2, 267-287.

[12] F. Pockels, Über die partielle Differentialgleichung $\Delta u+k^{2} u=0$, B. G. Teubner, Leipzig, 1891 (German).

[13] M. Práger, Eigenvalues and eigenfunctions of the Laplace operator on an equilateral triangle, Appl. Math. 43 (1998), no. 4, 311-320.

[14] G. F. Roach, Green's Functions, 2nd ed., Cambridge University Press, Cambridge, 1982.

[15] W. A. Strauss, Partial Differential Equations, An Introduction, John Wiley \& Sons, New York, 1992.

Brian J. McCartin: Department of Applied Mathematics, Kettering University, 1700 West Third Avenue, Flint, MI 48504-4898, USA

E-mail address: bmccarti@kettering.edu 


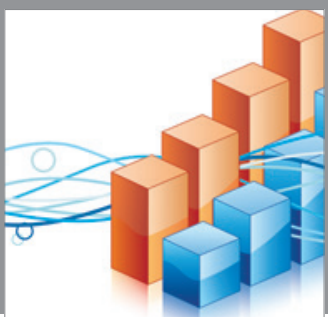

Advances in

Operations Research

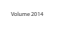

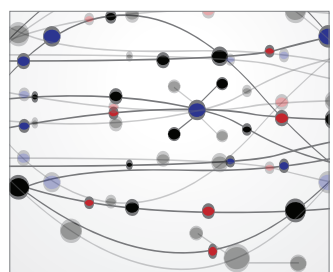

\section{The Scientific} World Journal
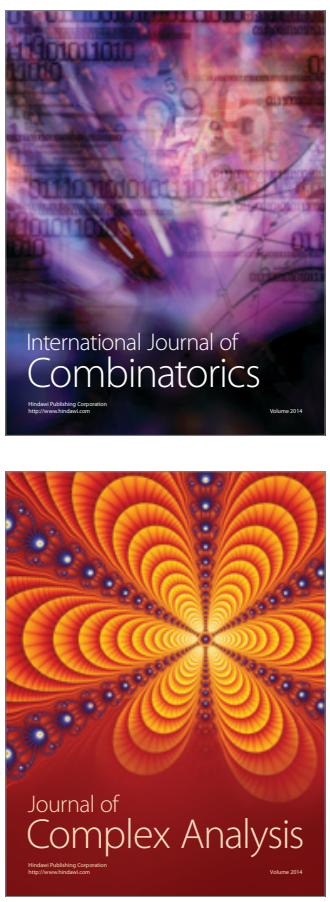

International Journal of

Mathematics and

Mathematical

Sciences
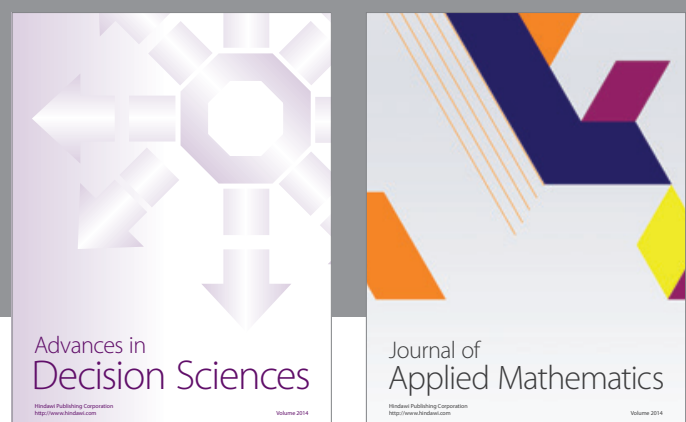

Journal of

Applied Mathematics
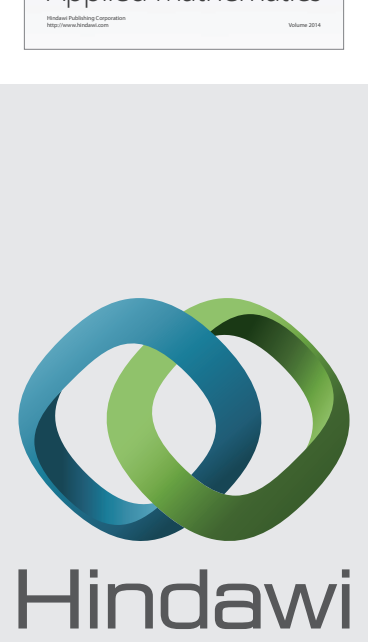

Submit your manuscripts at http://www.hindawi.com
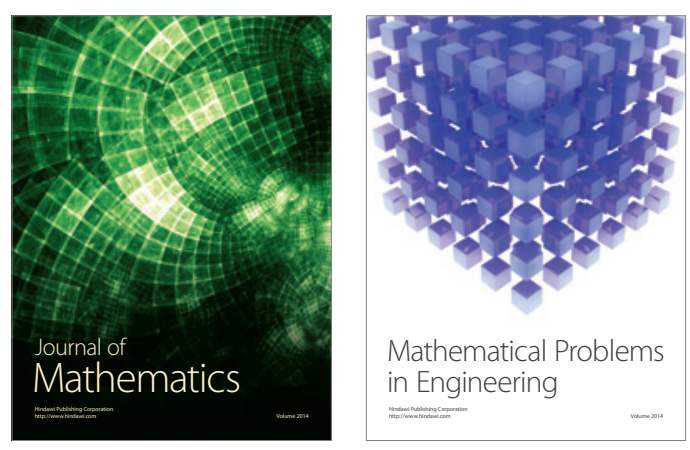

Mathematical Problems in Engineering
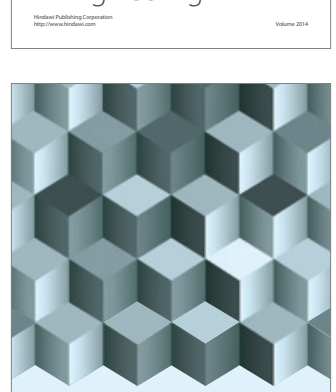

Journal of

Function Spaces
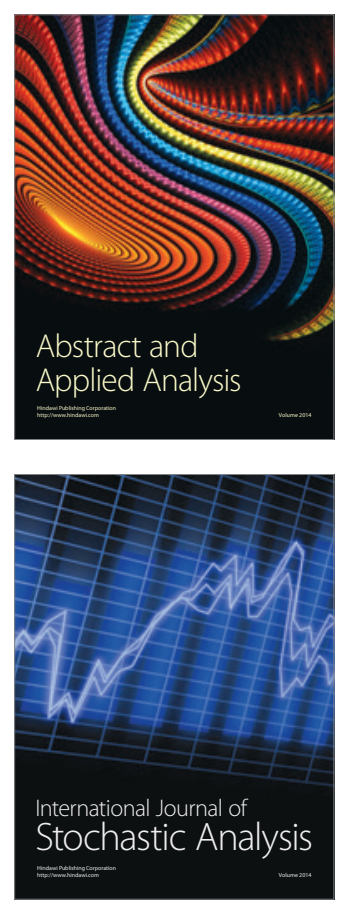

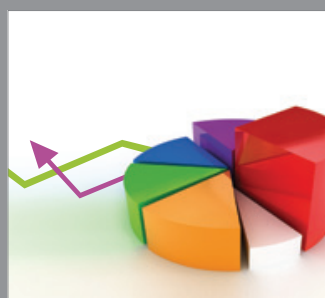

ournal of

Probability and Statistics

Promensencen
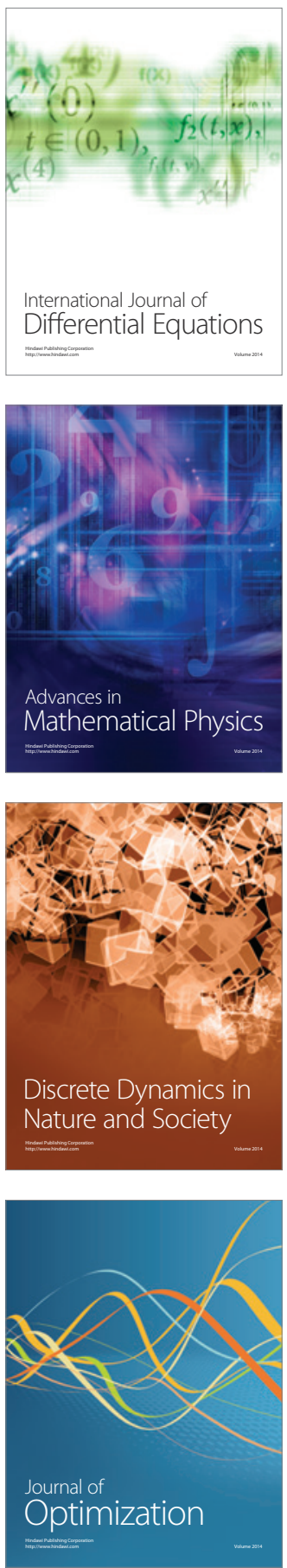